\title{
Influence of Electronic Structure on the Phase Diagram of Weakly Doped Superconducting Cuprates
}

\author{
A.M. Macia̧G AND P. WRÓBEL \\ Institute for Low Temperature and Structure Research \\ P.O. Box 1410, 50-950 Wrocław 2, Poland
}

Dedicated to Professor Józef Spatek on the occasion of his 60th birthday

\begin{abstract}
Motivated by the difference between the experimental phase diagram of cuprates and expectations of the $t-J$ model, we analyze the influence of the electronic structure on superconducting state generation for small levels of doping. Following some theoretical studies of the Fermi surface for hole doped superconducting cuprates we base our calculations on the $t-t^{\prime}-t^{\prime \prime}-J$ model. We construct the spin polaron model, which is an effective model for the $t-J$ model, and we expand it by adding the new terms related to the next neighbor hopping integrals $t^{\prime}$ and $t^{\prime \prime}$. In the framework of this model we study evolutions of superconducting correlation functions with doping. As a result of numerical calculations we find out that superconducting state vanishes for small levels of doping and finite values of $t^{\prime}$ and $t^{\prime \prime}$. This is in qualitative agreement with experimental results.
\end{abstract}

PACS numbers: 71.10.Fd, 74.20.Mn, 74.25.Dw

\section{Introduction and model}

It is well known that superconducting (SC) state is generated for an infinitesimally small amount of doping in the case of calculation done in the framework of the $t-J$ model $(t J \mathrm{M})$ on the square lattice $[1,2]$. On the other hand, the generation of the $\mathrm{SC}$ state is observed in $\mathrm{La}_{2}-\delta \mathrm{Sr}_{\delta} \mathrm{CuO}_{4}$ (LSCO) from $\delta$ about $5 \%$ [3]. This difference between theory and experiment can be related to the oversimplified picture of electronic structure. Namely, differences were also noticed between the Fermi surface measurements by means of angle-resolved photoelectron spectroscopy (ARPES) and calculations of the electronic structure made in the framework of the $t J \mathrm{M}$. This problem was solved by using the $t-t^{\prime}-t^{\prime \prime}-J$ model instead of the $t J \mathrm{M}$ in the calculations [4]. Following the idea mentioned before, 
we will use the $t-t^{\prime}-t^{\prime \prime}-J$ model to study the influence of electronic structure on the phase diagram of cuprates in the framework of the spin polaron model (SPM), which is an effective model for the $t J \mathrm{M}$ [5] on the square lattice. We assume that antiferromagnetic (AF) correlations, which are at least of short range, tend to confine a motion of holes created in the AF spin background. The nature of quasiparticle propagation and interaction between quasiparticles, which are called spin polarons is determined by a tendency to restore the local AF order. In the framework of the spin polaron model we define the wave function $\left|\Psi_{i}\right\rangle=\sum_{\mathcal{P}_{i}} \alpha_{\mathcal{P}_{i}}\left|\mathcal{P}_{i}\right\rangle$ of the spin polaron, at a given site $i$ as a combination of states $\left|\mathcal{P}_{i}\right\rangle$, which are created when a hole starting from the site $i$ hops along a path $\mathcal{P}_{i}$ in the empty AF ordered spin background. $\alpha_{\mathcal{P}_{i}}$ is defined as solutions of an approximate Schrödinger equation for the truncated $t J \mathrm{M}$ in the restricted basis spanning states $\left|\mathcal{P}_{i}\right\rangle$. The truncated Hamiltonian does not contain processes, which give rise to the hole deconfinement. These processes are considered when the effective Hamiltonian for the SPM is constructed. In the previous work [2] we constructed the effective Hamiltonian for the $t J \mathrm{M}$ in the language of spin polarons. In this paper we find new terms in SPM, which will appear as a result of adding the second nearest neighbor (SNN) hopping integral $t^{\prime}$ and the third nearest neighbor (TNN) hopping integral $t^{\prime \prime}$ to the $t J \mathrm{M}$. We will do this using the same method as in the case of the $t J \mathrm{M}$. First we analyze the single hole case. Our starting state represents a single hole, which is created on the site $i$ by the operator $c_{i, \uparrow}$ in the Néel state. This state is a component of spin polaron wave function with the amplitude $\alpha_{0}$, created on the same site. The state obtained by acting by means of the SNN hopping term on the starting state is also a component of the spin polaron wave function with the amplitude $\alpha_{0}$, but on the different site. The matrix element between these two states appears as the first new term of the one-particle part of effective Hamiltonian related with SNN hopping. This term is $\delta H_{1}=\frac{1}{2} t^{\prime} \alpha_{0}^{2} \sum_{\delta, \delta^{\prime}, \delta \perp \delta^{\prime}}\left(\sum_{i} h_{i+\delta+\delta^{\prime} \downarrow}^{\dagger} h_{i \downarrow}+\sum_{j} h_{j+\delta+\delta^{\prime} \uparrow}^{\dagger} h_{j \uparrow}\right)$. Fermionic operator $h_{i \downarrow}\left(h_{j \uparrow}\right)$ creates a hole on the site $i(j)$ located in the even (odd) sublattice in the AF ordered spin system. The next term is related to coupling of some states by the TNN hopping. It can be calculated in the same way as for SNN and takes the following form, $\delta H_{2}=t^{\prime \prime} \alpha_{0}^{2}\left(\sum_{i} h_{i+2 \delta \downarrow}^{\dagger} h_{i \downarrow}+\sum_{j} h_{j+2 \delta \uparrow}^{\dagger} h_{j \uparrow}\right)$. We will skip the detailed construction of other new terms, due to the lack of space, and list them below. $\delta H_{3}$ and $\delta H_{4}$ are related to the spin polaron pair propagation. The last three terms are related to the processes of decaying of such a pair, called a spin bipolaron, into two spin polarons and vice versa

$$
\delta H_{3}=t^{\prime}\left[\alpha_{0,0}^{2}+(z-2) \sum_{\mu=1}(z-1)^{\mu-1} \alpha_{0, \mu}^{2}\right]
$$




$$
\begin{aligned}
& \times \sum_{\delta, \delta^{\prime}, \delta^{\prime} \perp \delta}\left(\sum_{i} h_{i+\delta+\delta^{\prime} \downarrow}^{\dagger} h_{i+\delta \uparrow}^{\dagger} h_{i+\delta \uparrow} h_{i \downarrow}+\sum_{j} h_{j+\delta+\delta^{\prime} \uparrow}^{\dagger} h_{j+\delta \downarrow}^{\dagger} h_{j+\delta \downarrow} h_{j \uparrow}\right) \\
& \delta H_{4}=t^{\prime \prime}\left[\alpha_{0,0}^{2}+(z-2) \sum_{\mu=1}(z-1)^{\mu-1} \alpha_{0, \mu}^{2}\right] \\
& \times \sum_{\delta}\left(\sum_{i} h_{i+2 \delta \downarrow}^{\dagger} h_{i+\delta \uparrow}^{\dagger} h_{i+\delta \uparrow} h_{i \downarrow}+\sum_{j} h_{j+2 \delta \uparrow}^{\dagger} h_{j+\delta \downarrow}^{\dagger} h_{j+\delta \downarrow} h_{j \uparrow}\right), \\
& \delta H_{5}=t^{\prime}\left[\sum_{\mu=0}(z-1)^{\mu} \alpha_{\mu} \alpha_{0}\right] \sum_{\delta, \delta^{\prime}, \delta^{\prime} \perp \delta}\left[\sum_{i}\left(h_{i \downarrow}^{\dagger} h_{i+2 \delta+\delta^{\prime} \uparrow}^{\dagger} h_{i+\delta \uparrow} h_{i \downarrow}+\text { h.c. }\right)\right. \\
& \left.+\sum_{j}\left(h_{j \uparrow}^{\dagger} h_{j+2 \delta+\delta^{\prime} \downarrow}^{\dagger} h_{j+\delta \downarrow} h_{j \uparrow}+\text { h.c. }\right)\right] \\
& \delta H_{6}=t^{\prime}\left[\sum_{\mu=0}(z-1)^{\mu} \alpha_{\mu} \alpha_{0}\right] \sum_{\delta}\left[\sum_{i}\left(h_{i \downarrow}^{\dagger} h_{i+3 \delta \uparrow}^{\dagger} h_{i+\delta \uparrow} h_{i \downarrow}+\text { h.c. }\right)\right. \\
& \left.+\sum_{j}\left(h_{j \uparrow}^{\dagger} h_{j+3 \delta \downarrow}^{\dagger} h_{j+\delta \downarrow} h_{j \uparrow}+\text { h.c. }\right)\right] \\
& \delta H_{7}=t^{\prime}\left[\sum_{\mu=0}(z-1)^{\mu} \alpha_{\mu} \alpha_{0}\right] \sum_{\delta, \delta^{\prime}, \delta^{\prime} \perp \delta}\left[\sum_{i}\left(h_{i \downarrow}^{\dagger} h_{i+\delta+2 \delta^{\prime} \uparrow}^{\dagger} h_{i+\delta \uparrow} h_{i \downarrow}+\text { h.c. }\right)\right. \\
& \left.\left.+h_{j \uparrow}^{\dagger} h_{j+\delta+2 \delta^{\prime} \downarrow}^{\dagger} h_{j+\delta \downarrow} h_{j \uparrow}+\text { h.c. }\right)\right] .
\end{aligned}
$$

Now, we can use the complete SPM Hamiltonian for the $t-t^{\prime}-t^{\prime \prime}-J$ model to obtain the components of the SC order parameter. We will do it in the same way as we did in the case of the $t J \mathrm{M}[2]$.

\section{Numerical analysis}

Since the system which we analyze is two-dimensional, we concentrate on the case of vanishing temperature $T=0$ for which the SC state may exist. By minimizing the thermodynamic potential with respect to parameters $d \ldots$ defining the gap function and with respect to the chemical potential, we determine numerical values of these parameters. The gap function was defined in the real 


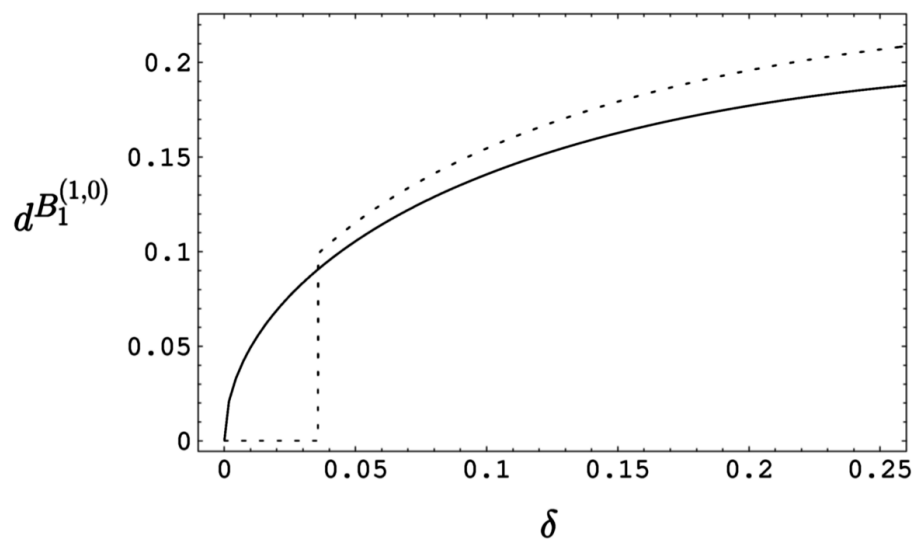

Fig. 1. Parameters $d_{1}^{B_{1}^{(1,0)}}$ which determine the SC correlation function defined in the real space at the distance of 1 lattice spacing as a function of the doping parameter $\delta$, calculated for parameters $t^{\prime} / t=-0.12, t^{\prime \prime} / t=0.08$ (dashed line). Solid line represents the case when the nearest neighbors hopping is considered the only one. $J / t=0.4$.

space on the distances $1, \sqrt{5}$, and 3 lattice constants. The results of the calculation are depicted by Fig. 1 . We consider two sets of parameters $t^{\prime} / t=0$, $t^{\prime \prime} / t=0$, and $t^{\prime} / t=-0.12, t^{\prime \prime} / t=0.08$. The second set has been chosen in order to describe the electronic structure of LSCO. The identification of the ratios $t^{\prime} / t$ and $t^{\prime \prime} / t$ based on the model analysis of ARPES spectra has been performed by Tohyama and Maekawa [4]. For each calculation we choose $J / t=0.4$. As it is depicted in Fig. 1, superconducting state is generated for a finite level of doping. For $t^{\prime} / t=-0.12, t^{\prime \prime} / t=0.08$ transition occurs for about $3 \%$ of doping, which is close to observed for LSCO. This transition is of the first order, which is probably related to the nature of the used approximation.

\section{Conclusions}

In the framework of SPM we show that the SC state is suppressed for the low level of doping in the case of parameters relevant for LSCO. One of the main reasons of this behavior is the possibility of propagation quasiparticles in the AF ordered spin system without the creation of defects in the magnetic structure. It turns out that the SPM describes the generation of the SC state for $\delta=0.03$, which is very close to the one observed in the case of LSCO for $\delta=0.05$. Our calculations show that the next nearest hopping modifies the phase diagram of the SC state which is generated in the weakly doped antiferromagnets. A further study of this phenomenon is required.

\section{Acknowledgments}

We thank R. Micnas for fruitful discussions. A.M. acknowledges the support of the State Committee for Scientific Research under contract No. 1P03B 05330. 


\section{References}

[1] S. Sorella, G.B. Martins, F. Becca, C. Gazza, L. Capriotti, A. Parola, E. Dagotto, Phys. Rev. Lett. 88, 117002 (2002).

[2] P. Wróbel, R. Eder, R. Micnas, J. Phys., Condens. Matter 15, 2775 (2003).

[3] A. Damascelli, Z.X. Shen, Z. Hussain, Rep. Math. Phys. 75, 473 (2003).

[4] T. Tohyama, S. Maekawa, Supercond. Sci. Techn. 13, R17 (2000).

[5] K.A. Chao, J. Spałek, A.M. Oleś, Phys. Rev. B 18, 3453 (1987). 